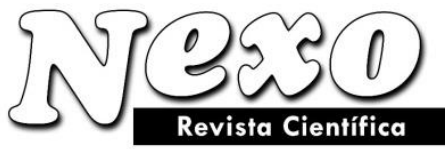

ISSN-E1995-9516

Universidad Nacional de Ingeniería COPYRIGHT @ (UNI). TODOS LOS DERECHOS RESERVADOS http://revistas.uni.edu.ni/index.php/Nexo https://doi.org/10.5377/nexo.v32i01.7986

Vol. 32, No. 01, pp. 41-51/Junio 2019

(c) $($ i) $($ ) $\odot$

\title{
FACTOR EROSIVIDAD DE LA LLUVIA EN LA SUBCUENCA SUR DEL LAGO XOLOTLÁN, MANAGUA
}

\section{RAINFALL EROSIVITY FACTOR IN THE XOLOTLÁN LAKE SUBCATCHMEN, MANAGUA}

\author{
M.E. Blanco Chávez \\ ${ }^{1}$ Universidad Nacional de Ingeniería. Managua, Nicaragua. \\ Programa de Investigación, Estudios Nacionales y Servicios del Ambiente. Managua, Nicaragua. \\ miguel.blanco@ piensa.uni.edu.ni
}

(recibido/received: 13-Diciembre-2018; aceptado/accepted:14-Mayo-2018)

\begin{abstract}
RESUMEN
En el presente artículo se aborda el estudio del índice de erosividad de la lluvia en la subcuenca sur del Lago Xolotlán que pertenece a la cuenca 9525 de Nicaragua. El objetivo del estudio fue la determinación del índice de erosividad $R$ de la Ecuación Universal Revisada de Pérdida de Suelo (RUSLE), a partir de los datos pluviográficos diarios de la estación 69027 (Aeropuerto de Managua) y datos pluviométricos mensuales de 7 estaciones distribuidas en la subcuenca y sus cercanías. Se analizaron los pluviogramas diarios del año 2007 al 2016, con los cuales se obtuvo los valores de $R$ mensual y anual. Se realizó una regresión lineal simple entre el valor de $R$ mensual y la precipitación mensual, con un coeficiente de correlación de $\mathrm{r}^{2}=0.86$. Dado que en la subcuenca de estudio sólo hay una estación pluviográfica, se utiliza el índice modificado de Fournier para estimar los valores de $R$ en las estaciones pluviométricas. El estudio sugiere que el índice promedio anual de la erosividad varía de 5,000 $\mathrm{Mj} \mathrm{mm} \mathrm{/} \mathrm{(ha-h-año)} \mathrm{en} \mathrm{la}$ parte baja de la subcuenca a 7,250 en la parte alta, representando la lluvia un poder erosivo moderado.
\end{abstract}

Palabras claves: Erosividad; Lluvia; Índice Modificado de Fournier; Índice de Concentración de la Precipitación.

\section{ABSTRACT}

In this paper the study of erosivity rainfall factor in the Xolotlán Lake sub catchment is exposed, that belong to the 9525 catchment from Nicaragua. The objective of the study was to determinate the Revised Universal Loss Equation (RUSLE) erosivity factor", from daily pluviograph data of the station 69027 (Managua Airport) and monthly pluviometric data in 7 stations distributed in the sub catchment and nearby. A simple lineal regression was done with the monthly $R$ and monthly precipitation, with a correlation coefficient of $r^{2}=0.86$. Since in the sub cathcment there is only one pluviograph station, the Modified Fournier Index was used to estimate de $R$ values in the pluviometrics stations. The study suggests that the annual mean erosivity factor rage from 5,000 Mj mm / (ha-h-year) in the lower side of the sub catchment to 7,250 in the upper side, that represent a rain with a moderate erosivity power.

Keywords: Erosivity; Rainfall; Modified Fournier index; Precipitation Concentration Index. 


\section{INTRODUCCIÓN}

La subcuenca sur del Lago Xolotlán pertenece a la cuenca 9525 (cuenca 69 en el sistema PHCA) o cuenca de los grandes lagos de Nicaragua. En ella se asientan importantes ciudades, entre ellas la ciudad capital Managua. La alta intervención humana en los recursos naturales de la subcuenca sur ha provocado importantes cambios en el uso del suelo y deterioro de los mismos, con fuertes procesos erosivos, tanto antropogénicos como naturales. El régimen pluviométrico en la subcuenca, con fuertes precipitaciones de corta duración en la época lluviosa contribuye grandemente a la erosión de la parte media y alta de la subcuenca en estudio, cuyos suelos de origen volcánico son susceptibles a ser rápidamente erosionados.

Con el fin de contribuir al desarrollo de herramientas para estimar la erosión, se ha realizado un estudio de la erosividad de la lluvia en la subcuenca sur del Lago Xolotlán. Para ello se han utilizado varios métodos de estimación, entre las que podemos mencionar la Ecuación Universal de Pérdida de Suelo, USLE por sus siglas en inglés, de Wischmeier y Smith (1978, pp. 3-4). Esta ecuación más tarde fue modificada por Renard et al (1997, p. 15) llamándola RUSLE. Estos autores determinaron el valore de " $R$ " en parcelas unitarias experimentales en los Estados Unidos. Otro método para estimar la erosividad es mediante regresiones lineales de " $R$ " con el Índice Modificado de Fournier. Se han realizado estudios para estimar “ $R$ ” con este método en países como Colombia (Rivera \& Gómez, 1991), España (Pérez \& Senent, 2015) y (M. de Luis, González, Raventos, Sánchez \& Cortina, 1997), Marruecos (Saldaña, Nemmaoui, Canton, \& Aguilar, 2014) y Estados Unidos (Renard \& Freimund, 1994). Dado que en la subcuenca sur del Lago Xolotlán hay pocos datos pluviográficos, se utilizó este método y por su sencillez de aplicación.

El desarrollo de esta metodología en la estimación del factor " $R$ ” permitirá aplicar el modelo RUSLE y hacer estimaciones de la erosión hídrica superficial en las zonas rurales de la subcuenca sur del Lago Xolotlán, poder hacer una mejor planificación del uso del suelo, tomar medidas de preservación del suelo y disminuir la erosión del suelo fértil del área rural.

Se plantea la hipótesis de que en la subcuenca sur del Lago Xolotlán se puede estimar con buena exactitud el factor erosividad de la lluvia " $R$ " en función del Índice Modificado de Fournier (IMF).

\section{MARCO TEÓRICO Y DESARROLLO}

\subsection{Estimación del factor de erosividad de la lluvia del método RUSLE.}

En base al análisis estadístico de los datos experimentales los investigadores Wischmeier y Smith (1978, pp. 3-4), en una publicación del Departamento de Agricultura de EUA presentan la Ecuación Universal de Pérdida de Suelo (USLE), que permite estimar la perdida promedio anual de suelo para un sitio específico bajo condiciones particulares de cultivos, tipo de suelo, pendiente y prácticas de cultivos. Este método fue ligeramente modificado por Renard at al (1997, pp. 14-18), del Departamento de Agricultura de EUA, llamándose Ecuación Universal Revisada de Pérdida de Suelos (RUSLE).

Factor erosividad de la lluvia-escorrentía (R): Los investigadores Wischmeier y Smith (1978, p. 5), aseguran que "los datos de investigación indican que, cuando todos los otros parámetros se mantienen constantes y sólo se varía la lluvia, las pérdidas de suelo por tormentas en campos cultivados son directamente proporcionales al parámetro de la tormenta identificado como "R =EI". El análisis de los datos (más de 30 años de mediciones en USA) adicionalmente demostró que el factor de la lluvia $R$, también deberá incluir los efectos acumulativos de muchas tormentas de tamaño moderado, así como también los efectos de las lluvias severas ocasionales.

El valor de $R$ para una tormenta (Wischmeier \& Smith, 1978, p. 5), es igual al producto de la energía total de la tormenta $(E)$ por la intensidad máxima de la tormenta en un período de 30 minutos $\left(I_{30}\right)$ :

Nexo Revista Científica / Vol. 32, No. 01, pp. 41-51/Junio 2019 
Donde:

$$
R=E I_{30}
$$

$R=$ índice de erosividad de la lluvia $(\mathrm{MJ} / \mathrm{ha}-\mathrm{mm} / \mathrm{h})$

$E=$ energía total de la tormenta $(\mathrm{MJ} / \mathrm{ha})$

$I_{30}=$ intensidad máxima de la tormenta en 30 minutos $(\mathrm{mm} / \mathrm{h})$

La relación de la pérdida de suelo con este parámetro es lineal, y los valores debido a tormentas individuales pueden ser sumados para obtener valores mensuales, estacionales y anuales. La suma de los valores de $E I$ de las tormentas de un período dado es una medida numérica del potencial erosivo de la lluvia en ese período.

El valor del índice de erosividad de la lluvia en un año, se estima sumando los valores de $R$ de todas las tormentas en ese año:

$$
R j=\sum_{i}^{n}\left(E I_{30}\right)_{i}
$$

Donde " $n$ " es el número de tormentas “ $i$ " en el año " $j$ "

El promedio total anual de los valores anuales de $E I$ de las tormentas en un sitio en particular es el índice de erosividad de la lluvia para ese sitio. El índice de erosividad promedio anual en un periodo de $N$ años es:

$$
R=\frac{\sum_{j}^{N} R_{j}}{N}
$$

La energía de la tormenta $E$ es un indicador del volumen y la escorrentía de esa tormenta, la intensidad $I_{30}$ indica la tasa de duración del pico de destrucción del suelo y escorrentía. El producto EI, técnicamente, indica cómo la desagregación de las partículas del suelo es combinada con la capacidad de transporte.

El valor de la energía cinética total $E$ de una tormenta se estima con:

$$
E=\sum_{k}^{m} e_{k} h_{k}
$$

Donde: " $e_{k}$ " es la energía cinética unitaria en el tramo $k$ de la lluvia (MJ/(ha-mm), " $h$ " es la altura de lluvia en el tramo " $k$ " en mm, " $m$ " es el número de tramos de la lluvia en estudio, y $E$ se expresa en $M J / h a$.

La energía de la tormenta es función de la cantidad de lluvia y de todas las intensidades componentes de la tormenta. Renard et al (1997, p. 25) citan a Brown y Foster (1987) quienes proponen estimar la energía cinética unitaria de la tormenta mediante la ecuación:

Donde:

$$
e=0.29[1-0.72 \exp (-0.05 I)]
$$

$e=$ energía cinética unitaria de un tramo " $k$ " de lluvia en Mega Julio por hectárea por milímetro de altura de lluvia del tramo (MJ/(ha-mm). 
$I=$ intensidad del tramo " $k$ " de la tormenta $(\mathrm{mm} / \mathrm{h})$.

Índice Modificado de Fourier (IMF): En un estudio realizado para la FAO en Marruecos Arnoldus (1980) plantea que "cuando no existe una adecuada densidad de estaciones pluviográficas para estimar el factor " $R$ " se puede utilizar una aproximación". En ese estudio presenta el índice modificado de Fournier como:

$$
I M F=\sum_{i=1}^{12} \frac{P_{i}^{2}}{P}
$$

Donde:

$P i=$ Lluvia promedio mensual $(\mathrm{mm})$

$P=$ Lluvia promedio anual $(\mathrm{mm})$

También demuestra que existe una alta correlación entre el factor de erosividad " $R$ ” y el $I M F$. Los resultados concuerdan muy bien con los valores de aquellas estaciones para las cuales el factor " $R$ " ha sido calculado con el método del RUSLE. Los resultados anteriores fueron confirmados por Renard y Freidmund (1994) en EUA con un alto coeficiente de correlación. El IMF ha sido utilizado en estudios para estimar " $R$ " en varios países como Colombia (Rivera \& Gómez, 1991), España (Pérez \& Senent, 2015), (De Luis, M., González, Raventos, Sánchez \& Cortina, 1997) y Marruecos (Saldaña, Nemmaoui, Canton \& Aguilar, 2014).

Índice de Concentración de la Precipitación (ICP): Los investigadores Lince y Castro (2015) citando a Oliver (1980) calculan la variabilidad temporal de la distribución de las lluvias en el año mediante el índice de concentración de las precipitaciones $(I C P)$ con:

$$
I C P_{j}=\frac{\sum_{i=1}^{12} P i^{2}}{\sum_{i=1}^{12}(P i)^{2}}
$$

Donde:

$P i=$ precipitación del mes i en el año $j(\mathrm{~mm})$.

$I C P=$ índice de concentración de la precipitación en el año $j(\%)$.

Los investigadores De Luis, González, Raventos, Sánchez y Cortina (1997) expresan "El índice se basa en la distribución mensual de la lluvia, por lo que con idénticas distribuciones mensuales de la misma el valor será idéntico para distintas cantidades. Por esta razón este índice valora la agresividad de la lluvia únicamente desde la perspectiva de la concentración”. Los límites teóricos van desde 8,3 si las lluvias en cada uno de los meses del año son similares, hasta 100 si todas las precipitaciones se concentran en un sólo mes (Tafur \& Obando, 2010).

\subsection{Metodología.}

Localización del área de estudio: La subcuenca sur del Lago Xolotlán pertenece a la cuenca 9525 (cuenca 69 en el sistema PHCA) o cuenca de los grandes lagos de Nicaragua (Figura 1) en la zona pacífica de Nicaragua. Tiene un área aproximada de $637 \mathrm{Km}^{2}$ y se encuentra entre los 11.95 a los 12.2 Latitud Norte, y $\operatorname{los} 86.076$ a los 86.408 Longitud Oeste. Los cursos de agua son efímeros, atraviesan la ciudad de Managua y drenan de sur a norte hasta desembocar en el Lago Xolotlán o de Managua. 


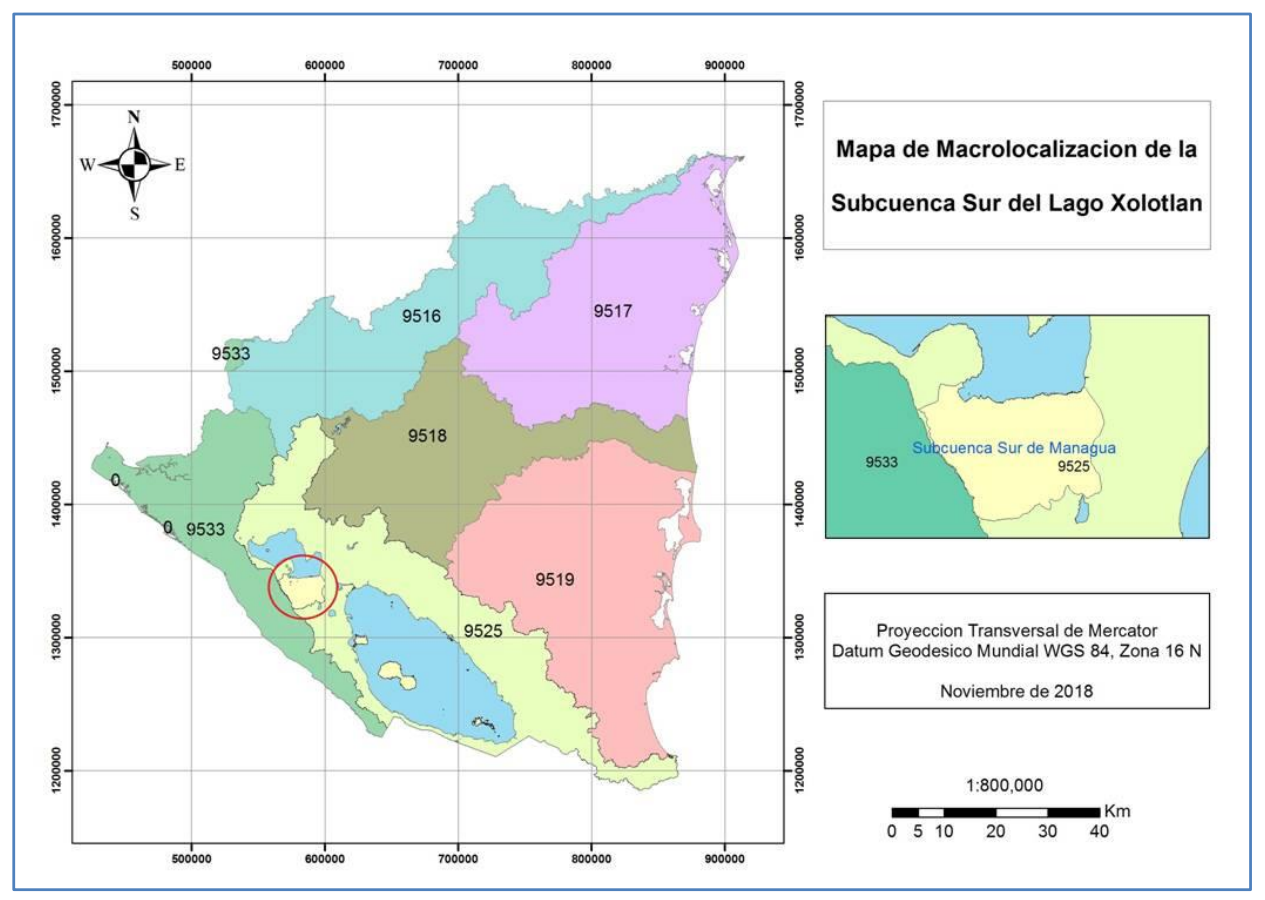

Figura 1. Localización de la Zona de estudio.

Información Pluviométrica: Se obtuvo del Instituto Nicaragüense de Estudios Territoriales (INETER) datos de precipitación de 6 estaciones meteorológicas, una de ellas con datos pluviográficos y cinco con únicamente datos pluviométricos. Los datos pluviográficos corresponden al período 2007-2016 (10 años) y los registros de datos pluviométricos varían en el rango 2001-2015, algunas estaciones todo el período y otras con menos registros. En la Tabla 1 se muestran las estaciones meteorológicas con registros pluviométricos utilizadas en este estudio y en la Figura 2 se muestra la ubicación de las mismas.

Tabla 1. Estaciones meteorológicas consideradas para el cálculo de la erosividad de la lluvia

\begin{tabular}{llccrrr}
\hline Código & Nombre de estación & Tipo & Latitud (N) & Longitud (W) & $\begin{array}{c}\text { Elevación } \\
(\mathrm{msnm})\end{array}$ & Años \\
\hline 69027 & AEROPUERTO ACS & HMP & $12^{\circ} 08^{\prime} 36^{\prime \prime}$ & $86^{\circ} 09^{\prime} 49^{\prime \prime}$ & 56 & $2001-2015$ \\
69089 & R.U.R.D. & HMP & $12^{\circ} 06^{\prime} 12^{\prime \prime}$ & $86^{\circ} 16^{\prime} 18^{\prime \prime}$ & 200 & $2001-2009$ \\
69104 & SAIMSA & HMO & $12^{\circ} 03^{\prime} 40^{\prime \prime}$ & $86^{\circ} 06^{\prime} 10^{\prime \prime}$ & 190 & $1993-2001$ \\
69115 & MASAYA (L. OXC.) & PV & $11^{\circ} 58^{\prime} 48^{\prime \prime}$ & $86^{\circ} 06^{\prime} 18^{\prime \prime}$ & 210 & $2001-2015$ \\
69046 & LA PRIMAVERA & PV & $12^{\circ} 01^{\prime} 08^{\prime \prime}$ & $86^{\circ} 14^{\prime} 55^{\prime \prime}$ & 600 & $2001-2012$ \\
69129 & CAMPOS AZULES & AG & $11^{\circ} 53^{\prime} 59^{\prime \prime}$ & $86^{\circ} 08^{\prime} 59^{\prime \prime}$ & 470 & $2001-2004$ \\
\hline Fuente: (INETER). & & & & & &
\end{tabular}

Información cartográfica: Se trabajo con un modelo de elevación del terreno (DEM) con tamaño de celda $30 \mathrm{~m}$ descargado de la página web del USGS y el shape de cuencas hidrográficas de Nicaragua delimitadas con la metodología Pfafstetter (INETER-UNI, 2014). 


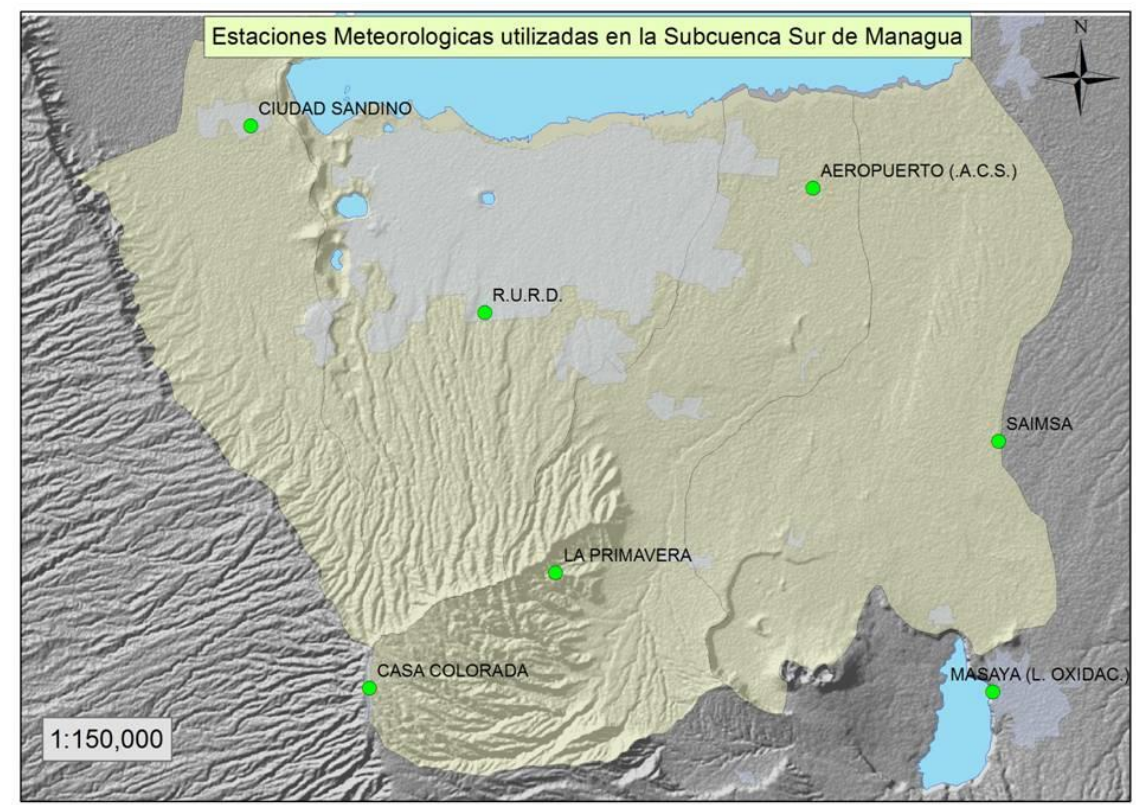

Figura 2. Localización de las estaciones meteorológicas utilizadas en el estudio

Procesamiento de datos: Los pluviogramas de lluvias diarias de la estación 69027 (Aeropuerto de Managua) en el período 2007-2016 fueron analizados para obtener las intensidades máximas en un período de 30 minutos, para los intervalos de un evento de lluvia con intensidad aproximadamente constante se obtuvieron la alturas precipitadas y las intensidades, así como la lluvia total del evento y la altura de lluvia total del día. Los datos de precipitación mensual de las estaciones pluviométricas fueron analizadas con la curva doble masa utilizando como estación índice la estación 69027, para comprobar la homogeneidad de sus registros, para las estaciones con datos faltantes se realizaron regresiones con otra estación vecina para el relleno de datos. En el caso de dos estaciones con registros muy cortos (la 69047 Cuidad Sandino y la 69171 Casa Colorada) se obtuvo sus datos de precipitación mensual del Atlas Climático de Nicaragua (INETER, 2004). En la Tabla 2 se presentan los valores de precipitaciones medias anuales procesadas para el periodo del estudio 2001-20015.

Tabla 2. Precipitaciones anuales (2001-2015) en las estaciones meteorológicas para el cálculo de la erosividad

\begin{tabular}{clcccc}
\hline Código & Nombre de estación & Tipo & $\begin{array}{c}\text { Elevación } \\
(\mathrm{msnm})\end{array}$ & Años & $\begin{array}{c}\text { P anual } \\
(\mathrm{mm})\end{array}$ \\
\hline 69027 & AEROPUERTO ACS & HMP & 56 & $2001-2015$ & 1136 \\
69089 & R.U.R.D. & HMP & 200 & $2001-2015$ & 1164 \\
69104 & SAIMSA & HMO & 190 & $2001-2015$ & 1084 \\
69115 & MASAYA (L. OXC.) & PV & 210 & $2001-2015$ & 1426 \\
69046 & LA PRIMAVERA & PV & 600 & $2001-2015$ & 1383 \\
69129 & CAMPOS AZULES & AG & 470 & $2001-2015$ & 1452 \\
69047 & CASA COLORADA & PV & 910 & $2001-2015$ & 1622 \\
69171 & CIUDAD SANDINO & PG & 100 & $2001-2015$ & 1090 \\
\hline
\end{tabular}

Índice modificado de Fournier: La estimación del índice modificado de Fournier (IMF) se realizo con (6) para todas las estaciones de precipitación consideradas en el estudio para el período 2001-20015. 
Índice de concentración de la precipitación: La estimación del índice de concentración de la precipitación $(I C P)$ se realizo con (7) para todas las estaciones de precipitación consideradas en el estudio para el periodo 2001-20015.

Índice de erosividad de la lluvia: La estimación del índice $R$ de un evento de lluvia se realizó para los pluviogramas de lluvias diarias de la estación 69027 (Aeropuerto de Managua) en el período 2007-2016, utilizando (1), correspondientes a $R$ del evento. Los valores de $R$ mensuales se obtuvieron sumando los valores de $R$ de los eventos correspondientes a ese mes. Los valores de $R$ anuales se obtuvieron con (2) y el valor de $R$ promedio anual se obtuvo con (3).

\subsection{Resultados y Análisis de Resultados}

Índice modificado de Fournier: Se estimó los valores del Índice Modificado de Fournier de las ocho estaciones meteorológicas. En la Tabla 3 se muestran los valores promedios interanuales del IMF. En general, utilizando el sistema de clasificación del $I M F$ propuesto por la Comisión de la Comunidad Europea (CEC, 1992), en la subcuenca sur los $I M F$ se clasifican como "Muy Altos" por tener valores mayores que 160, lo que nos indica que se obtendrán valores altos de índices de erosividad de la lluvia.

Índice de concentración de la precipitación: Se estimó los valores del Índice de Concentración de la Precipitación de las ocho estaciones meteorológicas. En la Tabla 3 se muestran los valores promedios interanuales de $I C P$. En general, utilizando el sistema de clasificación del ICP propuesto Michiels y Gabriels (1996), en la subcuenca sur los ICP se clasifican como "Estacional" por tener valores entre 16 y 20, lo que nos indica que las lluvias se concentran en una estación lluviosa, no son uniformes en todo el año, lo que incidirá con mayores valores de índices de erosividad de la lluvia.

Tabla 3. Valores promedios del IMF y del ICP de 8 estaciones meteorológicas de la subcuenca sur

\begin{tabular}{clcccc}
\hline Código & Nombre de estación & IMF & Clasificación & ICP $(\%)$ & Clasificación \\
\hline 69027 & AEROPUERTO ACS & 205 & Muy Alto & 18.2 & Estacional \\
69089 & R.U.R.D. & 198 & Muy Alto & 17 & Estacional \\
69104 & SAIMSA & 192 & Muy Alto & 17.8 & Estacional \\
69115 & MASAYA (L. OXC.) & 251 & Muy Alto & 17.6 & Estacional \\
69046 & LA PRIMAVERA & 243 & Muy Alto & 17.2 & Estacional \\
69129 & CAMPOS AZULES & 245 & Muy Alto & 16.8 & Estacional \\
69047 & CASA COLORADA & 225 & Muy Alto & 13.9 & M. Estacional \\
69171 & CIUDAD SANDINO & 167 & Muy Alto & 15.3 & M. Estacional \\
\hline
\end{tabular}

Índice de erosividad de la lluvia: Se estimaron los valores de $R$ para la estación pluviográfica del Aeropuerto de Managua (69027). La Tabla 4 muestra los valores estimados de $R$ anual y de los $I M F$ e ICP de la estación 69027 para el período 2007-2016. El valor más bajo de $R$ corresponde al año 2015, con un valor de $R=3885 \mathrm{Mj} \mathrm{mm} /$ (ha-h-año) y el más alto es $R=8267 \mathrm{Mj} \mathrm{mm} /$ (ha-h-año) en el año 2008 . 
M.E. Blanco Chávez

Tabla 4. Valores de $R$ anual, del IMF y del ICP de la estación 69027 (Aeropuerto de Managua)

\begin{tabular}{ccccc}
\hline Año & $\begin{array}{c}\text { P anual } \\
(\mathrm{mm})\end{array}$ & $I M F$ & $\begin{array}{c}I C P \\
(\%)\end{array}$ & $\begin{array}{c}R \text { anual } \\
\mathrm{Mj} \text { mm } /(\text { ha-h-año })\end{array}$ \\
\hline 2007 & 1412 & 229 & 16 & 6521 \\
2008 & 1440 & 287 & 20 & 8267 \\
2009 & 796 & 122 & 15 & 3418 \\
2010 & 1776 & 273 & 15 & 8216 \\
2011 & 1570 & 287 & 18 & 7711 \\
2012 & 1126 & 208 & 19 & 6146 \\
2013 & 1070 & 221 & 21 & 6036 \\
2014 & 826 & 164 & 20 & 4236 \\
2015 & 814 & 187 & 23 & 3881 \\
2016 & 986 & 185 & 19 & 6300 \\
Promedios & 1182 & 216 & 19 & 6073 \\
\hline
\end{tabular}

Una vez obtenidos los valores de $R$ anual e $I M F$ se procedió a realizar una regresión lineal de esas variables (Figura 3), con un nivel de significancia del $5 \%$ se obtuvo un coeficiente de correlación $\mathrm{r}^{2}=0.86$ para la siguiente ecuación:

$$
R=29.81 I M F-375.9
$$

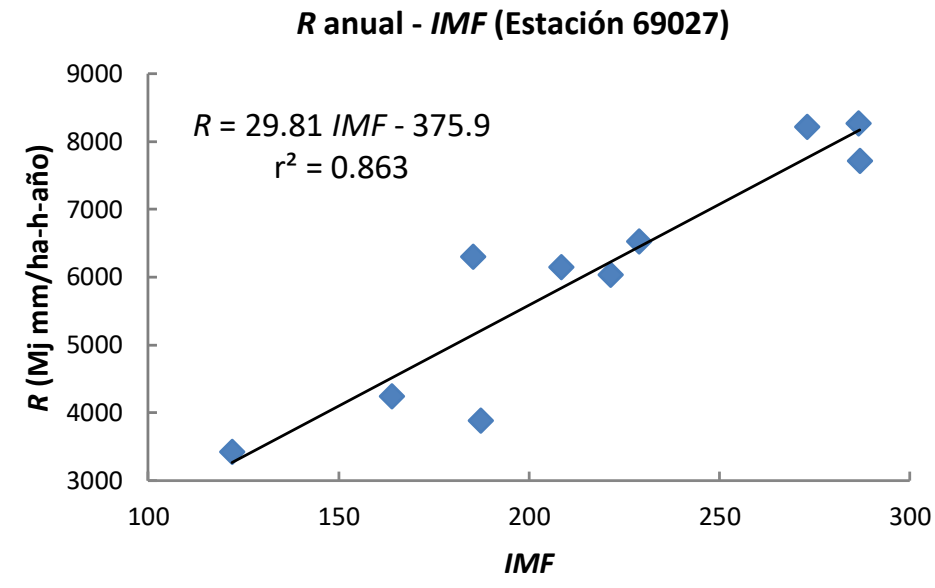

Figura 3. Correlación entre el factor $R(\mathrm{MJ} \mathrm{mm} /($ ha-h-año)) y el índice $I M F$, estación 69027.

La ecuación (8) nos permite estimar los valores de $R$ mensual en aquellas estaciones que únicamente tienen registros pluviométricos (no tienen pluviógrafo) y por tanto obtener también el valor de $R$ anual sumando los $R$ mensuales de cada año. Así, utilizando los valores $I M F$ de la Tabla 3 y (8), se estimaron los valores de $R$ de 7 estaciones pluviométricas de la subcuenca sur del Lago Xolotlán.

La Tabla 5 muestra los resultados anuales de este cálculo. Según la clasificación propuesta por Rivera y Gómez (1991), los valores de erosividad anual de la lluvia se clasifican como "Moderada" por estar entre 5,000 y 7,500 Mj mm / (ha-h-año), por lo que los procesos erosivos en la subcuenca sur son de consideración. 
M.E. Blanco Chávez

Tabla 5. Valores promedios anuales de $R$ en las 8 estaciones meteorológicas de la subcuenca sur

\begin{tabular}{clccc}
\hline Código & Nombre de estación & $I M F$ & $\begin{array}{c}R \text { anual } \\
\text { Mj mm/(ha-h-año) }\end{array}$ & $\begin{array}{c}\text { Clasificación } \\
\text { de } R\end{array}$ \\
\hline 69027 & AEROPUERTO ACS & 205 & 5720 & Moderada \\
69089 & R.U.R.D. & 198 & 5518 & Moderada \\
69104 & SAIMSA & 192 & 5357 & Moderada \\
69115 & MASAYA (L. OXC.) & 251 & 7092 & Moderada \\
69046 & LA PRIMAVERA & 243 & 6853 & Moderada \\
69129 & CAMPOS AZULES & 245 & 6913 & Moderada \\
69047 & CASA COLORADA & 225 & 6325 & Moderada \\
69171 & CIUDAD SANDINO & 167 & 4602 & Baja \\
\hline
\end{tabular}

Los valores anuales de $R$ de la Tabla 5 se utilizaron para obtener líneas de isoerosividad para la subcuenca sur del Lago Xolotlán. Para ello se utilizó el Software ArcMap, se interpolaron los valores de $R$ para obtener una capa raster de valores de $R$, y con ella se obtuvo las líneas de isoerosividad, como se muestra en la Figura 4.

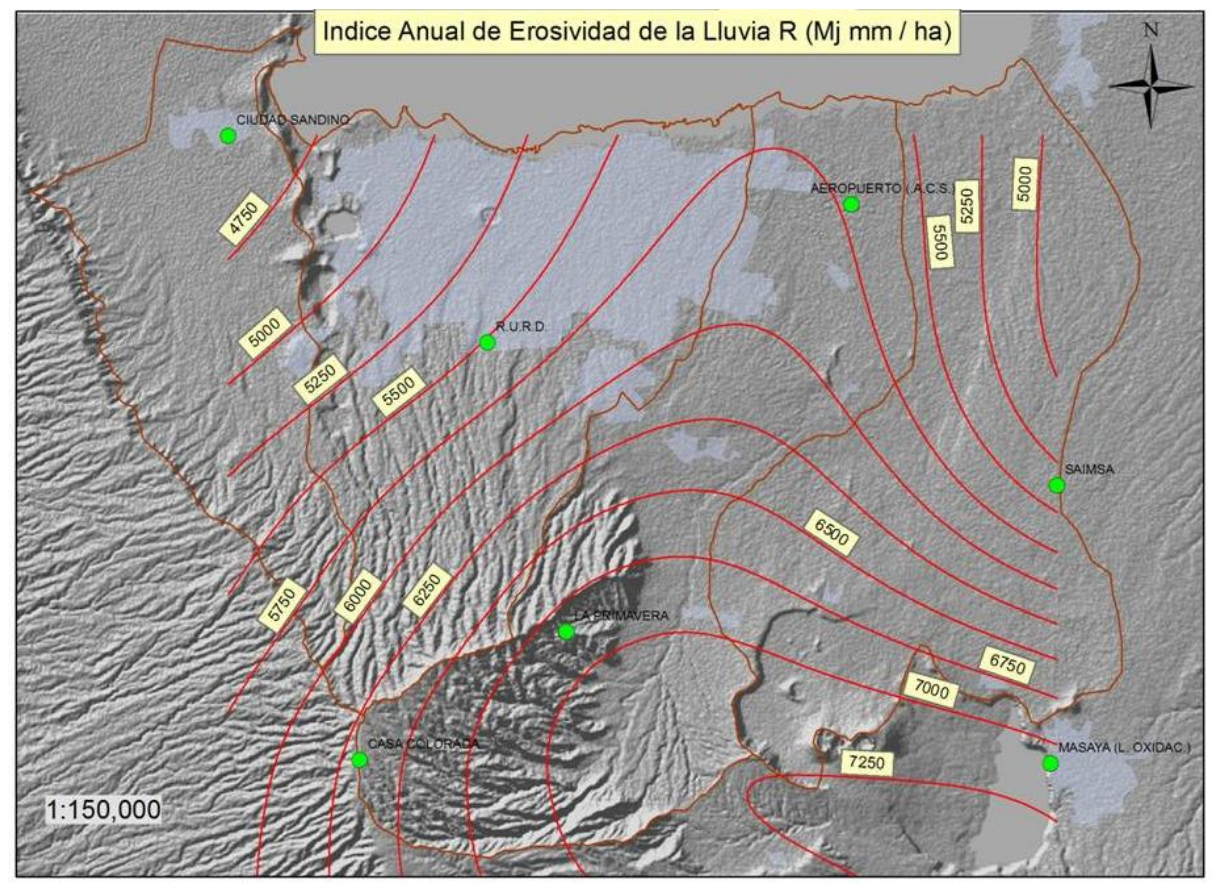

Figura 4. Índice de erosividad de la lluvia $R$ ( $\mathrm{Mj} \mathrm{mm} /$ (ha-h-año)

Se puede observar que los menores valores de erosividad de la lluvia se presentan en la parte baja de la subcuenca sur del Lago Xolotlan, que es la área más urbanizada, y que los mayores valores de erosividad de la lluvia se presentan en la parte alta de la subcuenca sur, que corresponde al área menos urbanizada con buen porcentaje de zonas de cultivos, y además, con mayores pendientes del terreno, por lo que podría provocar mayor erosión del suelo. Debe observarse que el mayor IMF de las estaciones pluviométricas que están en la parte alta de la cuenca, resultan en un mayor valor de $\mathrm{R}$ en la ecuación de correlación determinada. 


\section{CONCLUSIONES}

Se encontró buena correlación $\left(\mathrm{r}^{2}=0.86\right)$ entre el Índice Modificado de Fournier "IMF” y el factor de erosividad de la lluvia " $R$ " para los datos de la estación 69027 (Aeropuerto de Managua), mediante la ecuación $R=29.81(I M F)$ - 375.9, lo cual permite utilizar dicha relación para estimar el factor $R$ en las estaciones vecinas que sólo cuentan con datos pluviométricos.

El factor de erosividad de la lluvia tiene valores bajos en la parte norte de la subcuenca sur del Lago Xolotlán, en el área urbana, con valores entre 5,000 a 6,000 Mj mm / (ha-h-año), los valores más altos se presentan en la parte sur de la subcuenca, con valores entre 6,000 a 7,250 Mj mm / (ha-h-año).

Dado que la parte sur de subcuenca es zona rural, con áreas de cultivos y bosques, pendientes medias a altas, con suelos que se erosionan fácilmente y se presentan moderados valores de $R$, es necesario tomar medidas adecuadas de conservación de suelos para minimizar la acción erosiva de las lluvias, tales como el mantenimiento de una cobertura vegetal permanente sobre el suelo y no realizar labores de cultivo que involucren su remoción o exposición durante la estación lluviosa del año.

\section{REFERENCIAS}

Arnoldus, H.M.J. (1980). Methodology used to determine the maximum average soil loss due to sheet and rill erosion in Morocco. Food and Agriculture Organization of the United Nations Soils Bulletin, 34, 3948.

Commission of the European Communities (CEC). (1992). CORINE soil erosion risk and important land resources in the southern regions of the European Community.

De Luis, M., González-Hidalgo, J.C., Raventos, J., Sánchez, J.R., \& Cortina, J. (1997). Distribución espacial de la concentración y agresividad de la lluvia en el territorio de la Comunidad Valenciana. Cuaternario y Geomorfología, 11, 33-44.

Instituto Nicaragüense de Estudios Territoriales. (2004). Atlas Climático de Nicaragua. Managua.

Instituto Nicaragüense de Estudios Territoriales - Universidad Nacional de Ingeniería. (2014). Cuencas Hidrográficas de Nicaragua bajo la Metodología Pfafstter. Managua.

Lince, L., \& Castro, A. (2015). Erosividad de la lluvia en la zona cafetera del Quindío, Colombia. Cenicafe, $66 \quad$ (1), 25-31. $\quad$ Recuperado de http://biblioteca.cenicafe.org/bitstream/10778/606/1/arc066\%2801\%2925-31.pdf

Michiels, P., \& Gabriels, D. (1996). Rain variability indices for the assessment of rainfall erosivity in the Mediterranean region, 49-70. En: Soil degradation and desertification in Mediterranean environments. Geoforma editions'. Logroño, Spain: Rubio, J.L. and A. Calva (Eds.).

Pérez, J., \& Senent, J. (2015, octubre). Análisis comparativo de la evaluación de la erosividad de la lluvia en la cuenca del Guadalentín. IV Jornada de Ingeniería del Agua "La precipitación y los procesos erosivos". Córdoba, España.

Rivera P.,.J.H., \& Gómez, A.A. (1991). Erosividad de las lluvias en la zona cafetera central colombiana (Caldas, Quindío y Risaralda). Cenicafé, 42 (2), 37-52. 
Renard, K.G., \& Freimund, J.R. (1994). Using monthly precipitation data to estimate the R factor in the revised USLE. Journal of Hydrology, 157, 287-306.

Renard, K.G., Foster, G.A., Geesies, G.A., Mc Cool, D.K., \&Yoder, D.C. (1997). Predicting Soil Erosion by Water: A Guide to Conservation Planning with the Revised Universal Soil Loss Ecuation (RUSLE). Agriculture Handbook, 707. Washington, D.C.: U.S. Department of Agriculture (USDA).

Saldaña, M., Nemmaoui, A., Canton, M., Aguilar, M., \& Aguilar, F. (2014). Estimación de la erosión potencial en la cuenca del río Moulouya aguas arriba de la Presa Mohamed V. Mapping, 168, 4-16.

Soil Science Division Staff. (2017). Soil Survey Manual. Agriculture Handbook n . 18. Washington, D.C.: U.S. Department of Agriculture (USDA).

Tafur, L. E., \& Obando, F. H. (2010). Erosividad de las lluvias en la región Centro-Sur del Departamento de Caldas, Colombia. Facultad Nacional de Agronomía Medellín. 63 (1). Recuperado de https://revistas.unal.edu.co/index.php/refame/article/view/24951/36985

United States Deparment of Agriculture, Soil Conservation Service. (1971). Guides for interpreting Engineering Uses of soils. Washington, D.C.: U.S. Department of Agriculture (USDA).

Wischmeier, W. H., \& Smith, D. D. (1978). Predicting Rainfall Erosion Losses - A Guide to Conservation Planning. Agriculture Handbook, 537.Washington, D.C.: U.S. Department of Agriculture (USDA).

\section{SEMBLANZA DEL AUTOR}

Miguel E. Blanco Chávez: Obtuvo el grado de Ingeniero Civil en la Universidad Nacional de Ingeniería, Nicaragua. Desarrolló sus estudios de Maestría en Ingeniería Ambiental en el Programa de Investigación y Docencia en Medio Ambiente (PIDMA) de la UNI, Nicaragua. Ha participado como consultor en proyectos hidroeléctricos, de amenazas por inundación, de drenaje pluvial y manejo de cuencas hidrográficas. Ha sido profesor de hidráulica e hidrología en la carrera de ingeniería civil en la FTC de la UNI. Actualmente imparte los cursos Hidrología Aplicada y Redes de Alcantarillado en el Programa de Investigación, Estudios Nacionales y Servicios del Ambiente (PIENSA) de la UNI, Nicaragua. 